\title{
Aggregate QTca Interval
}

National Cancer Institute

\section{Source}

National Cancer Institute. Aggregate QT ca Interval. NCI Thesaurus. Code C124332.

The correction on the aggregate of QT intervals using individual probabilistic QT/RR slopes for each subject based on the measurement of QT intervals from multiple beats within a single ECG or from a defined time period within a continuous ECG. The method of aggregation, which can vary, is typically a measure of central tendency, such as mean. 